\title{
Optimal Management of Evolving Hierarchies
}

\author{
Hougaard, Jens Leth; Moreno-Ternero, Juan D.; Østerdal, Lars Peter
}

Document Version

Final published version

Publication date:

2018

\section{License \\ CC BY-NC-ND}

Citation for published version (APA):

Hougaard, J. L., Moreno-Ternero, J. D., \& Østerdal, L. P. (2018). Optimal Management of Evolving Hierarchies.

Department of Economics. Copenhagen Business School. Working Paper No. 11-2018

Link to publication in CBS Research Portal

\section{General rights}

Copyright and moral rights for the publications made accessible in the public portal are retained by the authors and/or other copyright owners and it is a condition of accessing publications that users recognise and abide by the legal requirements associated with these rights.

\section{Take down policy}

If you believe that this document breaches copyright please contact us (research.lib@cbs.dk) providing details, and we will remove access to the work immediately and investigate your claim. 
Copenhagen

Business School

HANDELSHøJSKOLEN

\section{Department of Economics}

Copenhagen Business School

Working paper 11-2018

\begin{tabular}{|c|}
\hline Optimal Management of Evolving \\
Hierarchies \\
Jens Leth Hougaard \\
Juan D. Moreno-Ternero \\
Lars Peter Østerdal \\
\end{tabular}

Department of Economics - Porcelænshaven 16A, 1. DK-2000 Frederiksberg 


\title{
Optimal Management of Evolving Hierarchies*
}

\author{
Jens Leth Hougaard ${ }^{\dagger}$ \\ Juan D. Moreno-Ternero \\ Lars Peter Østerdal ${ }^{\S}$
}

December 30, 2018

\begin{abstract}
We study the optimal management of evolving hierarchies, which abound in real-life phenomena. An initiator invests into finding a subordinate, who will bring revenues to the joint venture and who will invest herself into finding another subordinate, and so on. The higher the individual investment (which is private information), the higher the probability of finding a subordinate. A transfer scheme specifies how revenues are reallocated, via upward transfers, as the hierarchy evolves. Each transfer scheme induces a game in which agents decide their investment choices. We consider two optimality notions for schemes: initiator-optimal and socially-optimal schemes. We show that the former are schemes imposing to each member a full transfer to two recipients (the predecessor and the initiator) with a constant ratio among the transfers. We show that the latter are schemes imposing full transfers to the immediate predecessors.
\end{abstract}

JEL Classification: C70, L24, M52

Keywords: Optimal transfer schemes; Hierarchies; Management; Nash equilibrium

*We thank Jens Gudmundsson and audiences at National University of Singapore and University of Southern Denmark (GEM workshop) for valuable comments and suggestions on earlier versions of this paper. Financial support from the Independent Research Fund Denmark (DFF-6109-000132) and the Spanish Ministry of Economy and Competitiveness (ECO2017-83069-P) is gratefully acknowledged.

$\dagger$ Department of Food and Resource Economics, University of Copenhagen, Denmark.

$\ddagger$ Department of Economics, Universidad Pablo de Olavide, Spain.

$\S$ Department of Economics, Copenhagen Business School, Denmark. 


\section{Introduction}

It has been argued that the basic structure (or order) of the world is a hierarchical structure (e.g., Salthe, 1985). By a hierarchical structure (system) we mean, in general, an ensemble of interacting parts which is composed of successively nested sets of interacting subunits (e.g., Nicolis, 2010). Hierarchy seems a pervasive feature of the organization of natural and artificial systems (e.g., Corominas-Murtra et al., 2013). Hierarchical systems are frequent in large scale industrial automation such as the steel, petrochemical and electric power industries (e.g., Mesarovic et al., 1970). They also appear in countless scenarios we experience on a daily basis.

In this paper, we study the optimal management of evolving hierarchies. In our model, an initiator invests into finding a subordinate, who will bring revenues to the joint venture and who will invest herself into finding another subordinate. The higher the individual investment (which is private information), the higher the probability of finding a subordinate. Without investment, the probability reduces to zero. With large investments, the probability approaches one. The probabilistic process thus generates an evolving hierarchy.

A transfer scheme specifies how revenues are reallocated, via upward transfers, as the hierarchy evolves. Each transfer scheme induces a game in which agents decide their investment choices. The profile of investments and the transfer scheme determine the expected return from investments and the expected payoffs. The social value derived from the hierarchy is given by the expected total payoff. As transfers among agents cancel out, the transfer scheme is irrelevant for the computation of the optimal social value. Our first result is actually that the socially optimal investment profile is unique and constant among members of the hierarchy. In other words, the highest possible social value is obtained when all agents invest the same amount.

We then move to a decentralized framework and consider two optimality notions for schemes: initiator-optimal and socially-optimal schemes.

A transfer scheme is initiator optimal if it induces an equilibrium which yields the highest possible expected payoff for the initiator (achievable in equilibrium).

A transfer scheme is socially optimal if it induces an equilibrium which yields the highest possible social value (achievable in equilibrium).

We show that the initiator optimal schemes impose to each member a full transfer to only two recipients: the immediate predecessor and the initiator. Furthermore, a constant ratio among the transfers is imposed for all members. More precisely, there exists $\alpha \in(0,1)$ such 
that each agent transfers $\alpha$ of her revenues to her immediate predecessor and the rest to the initiator.

As for socially optimal schemes, we show that they simply impose full transfers to the immediate predecessors (and, therefore, no transfer to the initiator).

The rest of the paper is organized as follows. We finish the introduction referring to some related literature. We set the model in Section 2. We analyze the centralized management in Section 3. We deal in Sections 4 and 5 with the decentralized management (initiator optimality in the former and social optimality in the latter). In Section 6, we derive explicitly all the optimal schemes for a natural example. We conclude in Section 7. Some further computations have been gathered in an Appendix.

\subsection{Related literature}

Our model of managing endogenous hierarchies is somewhat related to a specific network intervention known in the literature as induction. The term network interventions describes the process of using social network data to accelerate behavior change or improve organizational performance (e.g., Valente, 2012). Induction interventions stimulate peer-to-peer interaction to create cascades in behavioral diffusion, implicitly endorsing that secondary incentives can be more efficient and effective than primary incentives, at least in some contexts. For instance, media marketing campaigns often rely on word-of mouth strategies, such as encouraging users to recommend products to their connections, who would do the same themselves (e.g., Aral and Walker, 2011). In respondent-driven sampling (e.g., Heckathorn, 1997), a form of chainreferral sampling also known as "snowball methods", individuals recruit others to receive an intervention, who subsequently encourage additional people to participate, and so on.

Goldlücke (2017) also deals with the strategic recruiting in ongoing hierarchies. Her emphasis is on analyzing the effect of skill-based promotions on incentives to recruit, exploring how the management rule "A's hire A's and B's hire C's" can make sense in a game-theoretic model.

Our analysis also focusses on the dichotomy between centralized and decentralized management of hierarchies. In that sense, we are close to a literature dealing with search and organizational hierarchy, in which it has been argued that a hierarchy with a central decision maker at the top can speed up problem solving, but possibly at the cost of solution quality compared with results of a decentralized search (e.g., Mihm et al., 2010; Rivkin, 2000). 
From a different vantage point, there has been a growing interest in the literature dealing with resource allocation in the presence of a hierarchical structure (see, for instance, Hougaard (2018) and the references cited therein). This interest can be traced back to Claus and Kleitman (1973) and Bird (1976), with their canonical cost sharing problem within a rooted tree, or Littlechild and Owen (1973) with the so-called airport problem, in which the runway cost has to be shared among different types of airplanes with a linear graph representing the runway. More recently, Hougaard et al., (2017) consider the problem of distributing the proceeds generated from a joint venture in which the participating agents are hierarchically organized. ${ }^{1}$ They characterize a family of allocation rules where revenue 'bubbles up' in the hierarchy, ranging from the no-transfer rule (where no revenue bubbles up) to the full-transfer rule (where all the revenues bubble up to the top of the hierarchy). Intermediate rules within that family are reminiscent of popular incentive mechanisms for social mobilization or multi-level marketing (e.g., Pickard et al., 2011; Emek et al., 2011) and can also be seen as specific geometric (incentive tree) mechanisms (e.g., Lv and Moscibroda, 2013) that are usually considered in the computer science literature.

\section{The benchmark model}

The set of potential agents is identified with the set of natural numbers including 0, i.e., $\mathbb{N}_{0}=\{0,1,2, \ldots\}$. We imagine a dynamic process where agent 0 represents the initial agent (to be thought of as the initiator, designer, boss, owner, patriarch, etc., depending on the application in mind) and starts the process to attract agent 1 as a follower. The probability of succeeding depends on how much resources agent 0 invests in the process. If agent 0 succeeds, this follower (agent 1) can now start the same process (as her predecessor) to attract agent 2 as a follower, and so forth, until some agent $(n)$ is unsuccessful in getting a follower. The outcome of the process would then be a realized hierarchy, representing an ordering of agents with higher numbers indicating a lower rank.

We assume that the ability of getting a follower is the same for every agent and is formalized by a function $p: \mathbb{R}_{+} \rightarrow[0,1)$, which assigns for each $x_{i} \in \mathbb{R}_{+}$, denoting agent $i$ 's search investment (i.e., the amount of resources that the agent has invested in the search), the probability $p\left(x_{i}\right) \in[0,1)$ that such an investment becomes successful. We refer to $p$ as the technology and

\footnotetext{
${ }^{1}$ This is reminiscent of the problem of sharing a polluted river (e.g., Ni and Wang, 2007; Dong et al., 2012), with the modification of considering negative revenues, and thus interpreting them as costs.
} 
assume that it is a strictly increasing, differentiable and strictly concave function, satisfying that $p(0)=0$, and $\lim _{x \rightarrow 0^{+}} p^{\prime}\left(x_{i}\right)=+\infty$. Note that we also assume $p\left(x_{i}\right)<1$, for each $x_{i} \in \mathbb{R}_{+}$. Thus, no matter how much a given agent invests in the search there is no guarantee of getting a follower. Also, we assume that agents' investment decisions are private information and that there is no budget constraint.

We assume that all agents joining the hierarchy are equally valuable and provide the same revenue, which we normalize to 1.

The initiator designs a transfer scheme specifying how revenues are reallocated as the hierarchy evolves. In line with the rank of the hierarchy we assume that revenues can only be transferred upwards in the realized hierarchy (that is, from agents with higher numbers to agents with lower numbers). A transfer scheme $t=\left\{t_{i j}\right\}_{\{i j\} \subset \mathbb{N}_{0}}$ is given by transfers $t_{i j} \in[0,1]$ such that $t_{i j}=0$ for each $i<j$ and $\sum_{j \leq i} t_{i j}=1$ for each $i$. Note that $t_{i j}$ is interpreted as the amount that agent $i$ has to transfer to agent $j$, provided $i$ joins the hierarchy.

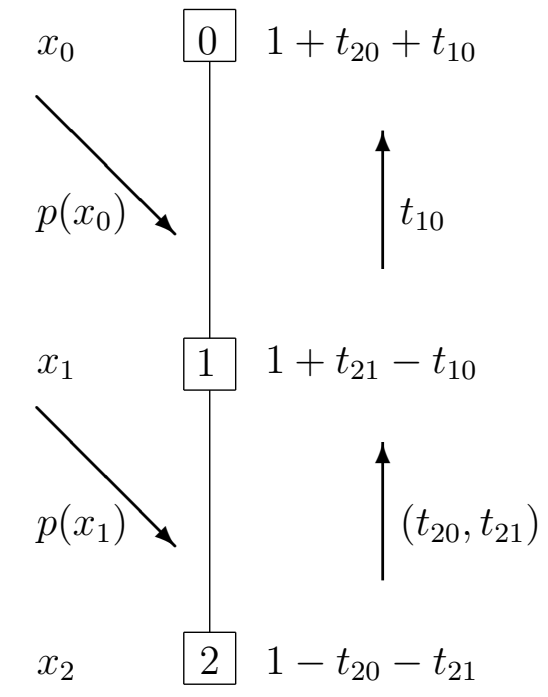

Figure 1: A realized hierarchy with three agents (the initiator and two successors).

For each agent $i \in \mathbb{N}_{0}$, each transfer scheme $t$, and each profile of investment choices $x=\left(x_{0}, x_{1}, x_{2}, \ldots\right)$, let $\mathbb{E}_{i}(t, x)$ denote the expected payoff for agent $i$ conditional on the realization of that agent. ${ }^{2}$ Formally,

$$
\mathbb{E}_{i}(t, x)=t_{i i}-x_{i}+\sum_{l=1}^{+\infty} \prod_{k=0}^{l-1} p\left(x_{i+k}\right) t_{(i+l) i} .
$$

We sometimes refer to the last term in (1), i.e., $\overline{\mathbb{E}}_{i}(t, x)=\sum_{l=1}^{+\infty} \prod_{k=0}^{l-1} p\left(x_{i+k}\right) t_{(i+l) i}$, as the gross expected return from investment.

\footnotetext{
${ }^{2}$ In principle, we allow for infinite expected payoffs.
} 
For any given profile of investments, $x$, the social value is given by the expected total payoff. Note that given a profile of investments, $x$, the transfer scheme $t$ is irrelevant for the computation of the social value because transfers among agents cancel out. Alternatively, one can write the social value as

$$
\mathbb{V}(x)=\left(1-x_{0}\right)+\sum_{i=1}^{+\infty} \prod_{k=0}^{i-1} p\left(x_{i+k-1}\right)\left(1-x_{i}\right) .
$$

We say that a profile of investments $x^{*}$ is first-best socially optimal, if the social value is maximized. As transfers are irrelevant, this kind of profile would be equivalently obtained analyzing a single-agent dynamic investment problem.

Note that first-best socially optimal profiles are dynamically consistent. By this we mean that they also maximize downward social value along the hierarchy. Formally, at any period $l$, the downward social value is given by

$$
\mathbb{V}^{l}\left(x_{l}, x_{l+1}, \ldots\right)=\left(1-x_{l}\right)+\sum_{i=1}^{+\infty} \prod_{k=l}^{i-1} p\left(x_{i+k-1}\right)\left(1-x_{i}\right)
$$

Then, if $x^{*}=\left(x_{0}^{*}, x_{1}^{*}, x_{2}^{*}, \ldots\right)$ is first-best socially optimal it follows that $x^{l *}=\left(x_{l}^{*}, x_{l+1}^{*}, \ldots\right)$ maximizes (3).

Each transfer scheme induces a game in which agents decide their investment choices (which are private information). A profile of investments $x^{*}=\left(x_{0}^{*}, x_{1}^{*}, \ldots\right)$ is a (Nash) equilibrium of this game if, for each agent $i \in \mathbb{N}_{0}$,

$$
\mathbb{E}_{i}\left(t,\left(x_{i}^{*}, x_{-i}^{*}\right)\right) \geq \mathbb{E}_{i}\left(t,\left(x_{i}, x_{-i}^{*}\right)\right),
$$

for each $x_{i} \in \mathbb{R}_{+}$.

In equilibrium, each agent $i \in \mathbb{N}_{0}$, if $x_{i}>0$, solves the following first order condition:

$$
p^{\prime}\left(x_{i}\right)=\frac{1}{t_{(i+1) i}+\sum_{l=2}^{+\infty} \prod_{k=1}^{l-1} p\left(x_{i+k}\right) t_{(i+l) i}} .
$$

Note that (4) shows that every agent has a unique best response given the other agents investment levels (equilibrium or not). In fact, the game is supermodular (Topkis, 1979) as there are monotonically increasing best replies.

As we shall argue later, agents' investments are, de facto, bounded and, thus, the fact that the game is supermodular means that for any technology, and any transfer scheme, there exists a pure strategy Nash equilibrium. 
Remark 1 The uniqueness of equilibria is not guaranteed. To show this, consider the technology defined by

$$
p\left(x_{i}\right)=\frac{\sqrt{x_{i}}}{\frac{1}{4}+\sqrt{x_{i}}} .
$$

Let $t$ be the transfer scheme such that $t_{i(i-2)}=1$, for each $i \geq 2, t_{00}=t_{11}=1$, and $t_{i j}=0$ otherwise. As $p(0)=0$, it follows that the profile $(0,0, \ldots)$ is an equilibrium. Now, (4) corresponds to

$$
1 / 8=\left(1 / 4+\sqrt{x_{i}}\right)^{3}
$$

which has the solution $x_{i}^{*}=1 / 16$, with $p\left(x_{i}^{*}\right)=1 / 2$. Thus, the profile $\left(x_{i}^{*}, x_{i}^{*}, \ldots\right)$ is also an equilibrium.

In the rest of the paper, we shall be mostly concerned with the two following decentralized optimality notions:

We say that a transfer scheme $t^{*}$ is (second-best) socially optimal if there exists a profile of investments $x^{*}$, which constitutes an equilibrium for the game induced by $t^{*}$, and $\mathbb{V}(\bar{t}, \bar{x}) \leq$ $\mathbb{V}\left(t^{*}, x^{*}\right)$, for each transfer scheme $\bar{t}$, inducing a game for which $\bar{x}$ is an equilibrium. Such a supporting equilibrium will sometimes be called socially-optimal equilibrium.

We say that a transfer scheme, $t^{*}$, is initiator-optimal if there exists a profile of investments $x^{*}$, which constitutes an equilibrium for the game induced by $t^{*}$, and $\mathbb{E}_{0}(\bar{t}, \bar{x}) \leq \mathbb{E}_{0}\left(t^{*}, x^{*}\right)$, for each transfer scheme $\bar{t}$, inducing a game for which $\bar{x}$ is an equilibrium. Such a supporting equilibrium will sometimes be called initiator-optimal equilibrium.

\section{Social optimality}

We concentrate in this section on the centralized approach to our model. As we shall see, the socially optimal investment profile is unique and constant among members of the hierarchy.

Our first result is about the existence of first-best socially optimal investment profiles.

Theorem 1 There exists a first-best socially optimal investment profile.

Proof. Let $x$ be a given investment profile. If $x$ is first-best socially optimal, the proof is finished. Otherwise, let $y^{1}=\left\{y_{i}^{1}\right\}$ be a profile such that $\mathbb{V}(x)<\mathbb{V}\left(y^{1}\right)$. Without loss of generality, we can assume that $y_{i}^{1} \leq 1$ for each $i=0, \ldots, \infty$. Indeed, if there exists an agent $i$ for which $y_{i}^{1}>1$, we define a new sequence $z^{1}$ for which $z_{k}^{1}=y_{k}^{1}, k=0, \ldots, i-1$ and if 
$\min \left\{j \mid j>i\right.$ and $\left.y_{j}^{1} \leq 1\right\} \neq \emptyset$, let $j^{*} \equiv \arg \min \left\{j \mid j>i\right.$ and $\left.y_{j}^{1} \leq 1\right\}$ and set $z_{i+k}^{1}=y_{j *+k}^{1}$ for $k=0, \ldots, \infty$. Otherwise, set $z^{1}=(0, \ldots, 0)$. In either case, $\mathbb{V}\left(z^{1}\right)>\mathbb{V}\left(y^{1}\right)$.

If $y^{1}$ is optimal, then the proof is finished. Otherwise, let $y^{2}=\left\{y_{i}^{2}\right\}$ be a profile such that $\mathbb{V}\left(y^{2}\right)>\mathbb{V}\left(y^{1}\right)$ and $y_{i}^{2} \leq 1$ for each $i=1, \ldots, \infty$. Eventually, we obtain a sequence of profiles $y^{k}=\left\{y_{i}^{k}\right\}$ such that $y_{i}^{k} \leq 1$ for each $i=1, \ldots, \infty$ and

$$
\mathbb{V}(x)<\mathbb{V}\left(y^{1}\right)<\ldots \mathbb{V}\left(y^{k-1}\right)<\mathbb{V}\left(y^{k}\right)<\ldots
$$

As $\mathbb{V}$ is a continuous function, and investments are bounded above by 1 , we know that

$$
\sup _{y} \mathbb{V}(y)<\infty
$$

We can therefore assume, without loss of generality, that we choose the sequence of profiles $y^{k}$ so that

$$
\lim \mathbb{V}\left(y^{k}\right)=\sup _{y} \mathbb{V}(y)<\infty .
$$

Now, as the sequence $y^{k}$ lies within the compact interval $[0,1]$, there exists a period-wise converging subsequence $\bar{y}^{k}$ of $y^{k}$, whose limit we denote by $\bar{y}^{*}=\lim _{k} \bar{y}^{k}$. By construction, $\bar{y}^{*}$ is first-best optimal.

Our second result is about the uniqueness of first-best socially optimal investments and its properties.

We say that a profile of investments $x$ is constant if $x_{i}=x_{j}$ for each pair $i, j \in\{1,2, \ldots\}$.

Theorem 2 The first-best socially optimal investment profile is unique and constant.

Proof. Let $\bar{x}$ be a first-best optimal profile of investments (which exists because of Theorem 1). Suppose, by contradiction, that $\bar{x}=\left(\bar{x}_{1}, \bar{x}_{2}, \ldots\right)$ is not constant. Then, there exists a pair $i, j \in\{1,2, \ldots\}$, such that $\bar{x}_{i} \neq \bar{x}_{j}$. As $\bar{x}$ is first-best socially optimal, it follows, by dynamic consistency, that

$$
\mathbb{V}^{i+1}\left(\bar{x}^{i+1}, \bar{x}^{i+2}, \ldots\right)=\mathbb{V}^{j+1}\left(\bar{x}^{j+1}, \bar{x}^{j+2}, \ldots\right)=\overline{\mathbb{V}}
$$

This means, in particular, that $\bar{x}_{i}$ and $\bar{x}_{j}$ are the solutions for the problems

$$
\arg \max p\left(x_{l}\right) \overline{\mathbb{V}}-x_{l},
$$

for $l=i, j$, whose first order conditions are

$$
p^{\prime}\left(x_{l}\right) \overline{\mathbb{V}}-1=0
$$


for $l=i, j$. Now, by the strict concavity of $p$, it follows that both solutions are the same, i.e., $\bar{x}_{i}=\bar{x}_{j}$, which is a contradiction. Uniqueness follows by an analogous argument.

In the next two sections, we turn to the decentralized approach to our model. First, we concentrate on initiator-optimal transfer schemes. Second, we concentrate on socially-optimal transfer schemes.

\section{Decentralized initiator optimality}

The next result characterizes the so-called initiator-optimal transfer schemes as those imposing to each member a full transfer to two recipients (the predecessor and the initiator) with a constant ratio among the transfers.

Theorem 3 There exists $\alpha \in(0,1)$ such that the scheme defined by $t_{(i+1) i}^{*}=\alpha, t_{i 0}^{*}=1-\alpha$, and $t_{i j}^{*}=0$ otherwise, is initiator-optimal.

\section{Proof.}

Step 1. There exists an initiator-optimal transfer scheme.

Theorem 1 implies that each agent's payoff and investment have a finite upper bound, which again implies that (without loss of generality) investments are bounded intervals. Now, take a sequence $\left(t^{k}, x^{k}\right)$ where $x^{k}$ is an equilibrium of the game induced by $t^{k}$. Choose the sequence such that it converges to $\sup _{(t, x)} \mathbb{E}_{0}(t, x)$, where $x$ is an equilibrium for the game induced by $t$.

Then, we can choose a subsequence such that, for each agent $i$, we have that $x_{i}^{k}$ converges to $\bar{x}_{i}$ and $t_{i j}^{k}$ converges to $\bar{t}_{i j}$. Thus, we obtain that $(\bar{t}, \bar{x})$ is an equilibrium (as each agent's payoff is continuous in $t$ and $x$ ), from where we conclude that $\bar{t}$ is an initiator-optimal transfer scheme.

Step 2. There exists $\alpha \in(0,1)$ such that the scheme defined by $t_{(i+1) i}^{*}=\alpha, t_{i 0}^{*}=1-\alpha$, and $t_{i j}^{*}=0$ otherwise is initiator-optimal

Let $t$ be an initiator-optimal transfer scheme. We now claim some properties of $t$.

First, $t_{i i}=0$, for each $i \in \mathbb{N}_{0} \backslash\{0\}$. The reason is simply that a self-transfer is irrelevant for the investment decision. More precisely, if $t_{i i}>0$, for some $i \in \mathbb{N}_{0} \backslash\{0\}$, and $x$ is an equilibrium of the induced game, then we could construct a transfer $t^{\prime}$, such that $t_{i i}^{\prime}=0, t_{i 0}^{\prime}=t_{i 0}+t_{i i}$, and $t_{j k}^{\prime}=t_{j k}$ otherwise, and whose induced game would have $x^{\prime}$ as an equilibrium, such that $\mathbb{E}_{0}(t, x)<\mathbb{E}_{0}\left(t^{\prime}, x^{\prime}\right)$ 
Let $N_{i}^{+}$denote the set of $i$ 's followers (including $i$ ) in the hierarchy, i.e., $N_{i}^{+}=\{i, i+1, \ldots\}$. Let $x$ be an initiator-optimal equilibrium in the game induced by $t$, and let $\mathbb{S}_{i}(t, x)$ denote the expected total transfers from members of coalition $N_{i}^{+}$to the predecessors of $i$, given agent $i$ joins the hierarchy. Formally,

$$
\mathbb{S}_{i}(t, x)=\sum_{j=i}^{+\infty} \prod_{l=0}^{j-1} p\left(x_{l}\right) \sum_{k=0}^{i-1} t_{j k} .
$$

Let $j$ be such that $0<j<i$. We claim that $\mathbb{S}_{i}(t, x)=\mathbb{S}_{j}(t, x)$.

Suppose, by contradiction, that $\mathbb{S}_{i}(t, x)>\mathbb{S}_{j}(t, x)$. Then, agent 0 can design a new transfer scheme $t^{\prime}$ in which the transfers of $N_{i}^{+}$are copied onto $N_{j}^{+}$, i.e., $t_{k l}^{\prime}=t_{(k+(i-j)) l}$, for each $k \in N_{j}^{+}$. Moreover, for each $k \notin N_{j}^{+}, t_{k l}^{\prime}$ is such that $\mathbb{E}_{k}(t, x)=\mathbb{E}_{k}\left(t^{\prime}, x\right)$. Any residual revenue goes to agent 0 . Note that such a transfer scheme $t^{\prime}$ is feasible due to the hypothesis that $\mathbb{S}_{i}(t, x)>\mathbb{S}_{j}(t, x)$.

As $x$ was an initiator-optimal equilibrium of the game induced by $t$, the transfer scheme $t^{\prime}$ induces an equilibrium $x^{\prime}$ with the following features:

- $x_{0}^{\prime}>x_{0}$ and $\mathbb{E}_{0}\left(t^{\prime}, x^{\prime}\right)>\mathbb{E}_{0}(t, x)$,

- $x_{l}^{\prime}=x_{l}$ for each $l=1,2, \ldots, j-1$,

- $x_{l}^{\prime}=x_{i+j-l}$ for each $l=j, j+1, \ldots$

But then, because of the first item, $t$ is not initiator-optimal, which represents a contradiction.

Suppose now that $\mathbb{S}_{i}(t, x)<\mathbb{S}_{j}(t, x)$. Then agent 0 can design a new transfer scheme $t^{\prime}$ in which the transfers of $N_{j}^{+}$are copied onto $N_{i}^{+}$, i.e., $t_{k l}^{\prime}=t_{(k+(j-i)) l}$, for each $k \in N_{i}^{+}$. Moreover, for each $k \notin N_{i}^{+}, t_{k l}^{\prime}$ is such that $\mathbb{E}_{k}(t, x)=\mathbb{E}_{k}\left(t^{\prime}, x\right)$. Any residual revenue goes to agent 0 . Note that such a transfer scheme $t^{\prime}$ is feasible due to the hypothesis that $\mathbb{S}_{i}(t, x)<\mathbb{S}_{j}(t, x)$.

As $x$ was an initiator-equilibrium of the game induced by $t$, the transfer scheme $t^{\prime}$ induces an equilibrium $x^{\prime}$ with the following features:

- $x_{0}^{\prime}>x_{0}$ and $\mathbb{E}_{0}\left(t^{\prime}, x^{\prime}\right)>\mathbb{E}_{0}(t, x)$,

- $x_{l}^{\prime}=x_{l}$ for each $l=1,2, \ldots, i-1$,

- $x_{l}^{\prime}=x_{l-i+j}$ for each $l=i, i+1, \ldots$.

But then, because of the first item, $t$ is not initiator-optimal, which represents a contradiction.

We therefore conclude that $\mathbb{S}_{i}(t, x)=\mathbb{S}_{j}(t, x)$. 
Next, we argue that an initiator-optimal transfer scheme induces a symmetric equilibrium among the agents following the initiator. For this, suppose that $t$ is initiator-optimal and $x$ is a corresponding boss optimal equilibrium.

Now, suppose that for some $i \geq 1$ we have $x_{i} \neq x_{i+1}$.

We will argue that it is then possible to change $t$ to another transfer scheme $\hat{t}$ such that a new equilibrium $\hat{x}$ is obtained with $\hat{x}_{i}=\hat{x}_{i+1}$ and $\hat{x}_{j}=x_{j}$ for all $j \leq i$, and in particular the payoff to agent 0 is the same at $\hat{x}$ than at $x$.

For this, we will establish $\hat{t}$ such that agent $i+1$ faces the same transfers from subsequent agents as $i$ did under scheme $t$. More precisely, let $\hat{t}$ satisfy the following:

- $\hat{t}_{k j}=t_{(k-1)(j-1)}$, for each $k \geq j \geq i+1$,

- $\hat{t}_{k j}=t_{k j}$, for each $j \leq k \leq i+1$,

- $\sum_{j=0}^{i} \hat{t}_{k j}=\sum_{j=0}^{i-1} t_{(k-1) j}$, for each $k \geq i+2$ and each $j=0, \ldots, i$.

Now, let $\hat{x}$ be defined by $\hat{x}_{j}=x_{j}$ for $j \leq i$ and $\hat{x}_{j}=x_{j-1}$ for $j>i$. Then, as $S_{i+1}(\hat{t}, \hat{x})=$ $S_{i}(t, x)$ it is possible to choose the transfers $\hat{t}_{k j}$ (for $j \leq i, k \geq i+2$ ) such that each agent $j \leq i$ gets the same total expected payoff from investing in a follower under $\hat{t}$ as under $t$. Thus, $\hat{x}$ is an equilibrium under $\hat{t}$, and we are done.

Starting at $i=1$ this process can be sequentially repeated for increasing $i$.

Now, let $\alpha=\overline{\mathbb{E}}_{i}(t, x)>0$. Note that the highest possible revenue obtained from the hierarchy is $\sum_{n=0}^{\infty}(p(x))^{n}=\frac{1}{1-p(x)}$. On the other hand, the overall gross expected return would be $\sum_{n=0}^{\infty}(p(x))^{n} \alpha=\frac{\alpha}{1-p(x)}$. By feasibility, it follows that $\alpha<1$.

To conclude, consider the scheme $t^{*}$ defined by $t_{(i+1) i}^{*}=\alpha, t_{i 0}^{*}=1-\alpha$, and $t_{i j}^{*}=0$ otherwise. This scheme induces a game with a symmetric equilibrium with strictly positive investments in which each agent (except for 0) gets a gross expected return from investment of $\alpha$. Thus, 0 gets the residual, i.e., $(1-\alpha) \sum_{n=0}^{\infty}(p(x))^{n}=\frac{1-\alpha}{1-p(x)}$.

The previous result characterizes the initiator-optimal transfer schemes as those imposing to each member a full transfer to two recipients (the predecessor and the initiator) with a constant ratio among the transfers. But its proof is silent about the construction of the optimal ratio $(\alpha)$ describing the corresponding optimal scheme. It turns out that it is the solution to the problem

$$
\max _{\alpha \in[0,1]} \Phi(\alpha),
$$


where $\Phi:[0,1] \rightarrow \mathbb{R}$ is such that, for each $\alpha \in[0,1]$

$$
\Phi(\alpha)=\left(1-\bar{x}_{\alpha}\right)+p\left(\bar{x}_{\alpha}\right)\left(\frac{1-\alpha p\left(x_{\alpha}^{*}\right)}{1-p\left(x_{\alpha}^{*}\right)}\right)
$$

and $x_{\alpha}^{*}$ is such that

$$
\alpha p^{\prime}\left(x_{\alpha}^{*}\right)=1
$$

whereas $\bar{x}_{\alpha}$ is such that

$$
p^{\prime}\left(\bar{x}_{\alpha}\right)=\frac{1-p\left(x_{\alpha}^{*}\right)}{1-\alpha p\left(x_{\alpha}^{*}\right)} .
$$

Note that (7) guarantees that all followers are selecting their optimal investment, given the scheme $t^{\alpha}$, whereas (8) guarantees that the initiator is selecting her optimal investment, given the scheme $t^{\alpha}$. Finally, (6) reflects the expected payoff for the initiator, at those investment choices, i.e.,

$$
\Phi(\alpha)=\mathbb{E}_{0}\left(t^{\alpha},\left(\bar{x}_{\alpha}, x_{\alpha}^{*}, x_{\alpha}^{*}, \ldots\right)\right)
$$

\section{Decentralized social optimality}

We now focus on (second-best) socially optimal transfer schemes. The next result characterizes them as those imposing full transfers to the immediate predecessors.

Theorem 4 The scheme defined by $t_{(i+1) i}=1$, for $i=0, \ldots$, and $t_{i j}=0$ otherwise, is decentralized socially optimal.

Proof. Given investment profile $x$, define the expected length of the hierarchy as $\mathbb{L}(x)=$ $1+\sum_{i=1}^{+\infty} \prod_{k=0}^{i-1} p\left(x_{k}\right)$. For a given expected investment $\mathbb{X}$, let $\mathbb{L}^{*}(\mathbb{X})$ be the highest obtainable expected length from an investment profile with expected investment $\mathbb{X}$.

Step 1. $\mathbb{L}^{*}$ is a strictly concave function.

Let $x$ and $y$ be two investment profiles inducing expected investments $\mathbb{X}$ and $\mathbb{Y}$, respectively. Consider a fair lottery between $x$ and $y$, denoted by $\bar{z}=\left[\left(x ; \frac{1}{2}\right),\left(y ; \frac{1}{2}\right)\right] .^{3}$ Then, the expected investment of $\bar{z}$ is given by $\frac{1}{2} \mathbb{X}+\frac{1}{2} \mathbb{Y}$ and its expected length is given by $\frac{1}{2} \mathbb{L}(x)+\frac{1}{2} \mathbb{L}(y)$. We aim to show that, based on this lottery, we can derive an investment profile $z$ with expected investment equal to $\frac{1}{2} \mathbb{X}+\frac{1}{2} \mathbb{Y}$ and expected length strictly larger than $\frac{1}{2} \mathbb{L}(x)+\frac{1}{2} \mathbb{L}(y)$.

\footnotetext{
${ }^{3}$ We endorse the standard notation for lotteries. Thus, $[(x ; p),(y ; 1-p)]$ indicates that profile $x$ occurs with probability $p$, whereas profile $y$ occurs with probability $1-p$.
} 
For each $t=1,2, \ldots$, let $x^{\mid t}=\left(x_{t}, x_{t+1}, \ldots\right)$ and $y^{\mid t}=\left(y_{t}, y_{t+1}, \ldots\right)$. Now, define the lottery

$$
\hat{z}=\left[\left(\frac{1}{2} x_{0}+\frac{1}{2} y_{0}, x^{\mid 1} ; s_{1}\right),\left(\frac{1}{2} x_{0}+\frac{1}{2} y_{0}, y^{\mid 1} ; s_{2}\right),\left(\frac{1}{2} x_{0}+\frac{1}{2} y_{0}, 0^{\mid 1} ; 1-s_{1}-s_{2}\right)\right],
$$

where $s_{1} \in[0,1]$ and $s_{2} \in[0,1]$ satisfy that

$$
\frac{1}{2} p\left(x_{0}\right)=p\left(\frac{1}{2} y_{0}+\frac{1}{2} x_{0}\right) s_{1}, \text { and } \frac{1}{2} p\left(y_{0}\right)=p\left(\frac{1}{2} y_{0}+\frac{1}{2} x_{0}\right) s_{2} .
$$

Note that, by the concavity of $p, p\left(\frac{1}{2} y_{0}+\frac{1}{2} x_{0}\right) \geq \frac{1}{2} p\left(y_{0}\right)+\frac{1}{2} p\left(x_{0}\right)$. Thus, $s_{1}+s_{2} \leq 1$.

That is, $\hat{z}$ is the lottery indicating that, with probability $s_{1}$, the investment profile $x$ materializes, with the exception of having $\frac{1}{2} x_{0}+\frac{1}{2} y_{0}$ for the initiator's investment, instead of $x_{0}$. Likewise, with probability $s_{2}$, the investment profile $y$ materializes, with the exception of having $\frac{1}{2} x_{0}+\frac{1}{2} y_{0}$ for the initiator's investment, instead of $y_{0}$. Finally, with probability $1-s_{1}-s_{2}$, the investment profile 0 materializes, with the exception of having $\frac{1}{2} x_{0}+\frac{1}{2} y_{0}$ for the initiator's investment, instead of 0 .

Note also that $s_{1}$ and $s_{2}$ are chosen so that the expected investment of $\hat{z}$ is equal to that of $\bar{z}$, i.e., $\frac{1}{2} \mathbb{X}+\frac{1}{2} \mathbb{Y}$. Now,

$$
\mathbb{L}(\bar{z})=\frac{1}{2}\left(1+p\left(x_{0}\right)+p\left(x_{0}\right) p\left(x_{1}\right)+\ldots\right)+\frac{1}{2}\left(1+p\left(y_{0}\right)+p\left(y_{0}\right) p\left(y_{1}\right)+\ldots\right)=\frac{1}{2} \mathbb{L}(x)+\frac{1}{2} \mathbb{L}(y),
$$

and

$$
\mathbb{L}(\hat{z})=1+p\left(\frac{1}{2} y_{0}+\frac{1}{2} x_{0}\right)+p\left(\frac{1}{2} y_{0}+\frac{1}{2} x_{0}\right)\left(s_{1} p\left(x_{1}\right)+s_{2} p\left(y_{1}\right)\right)+\cdots>\frac{1}{2} \mathbb{L}(x)+\frac{1}{2} \mathbb{L}(y),
$$

as desired.

Step 2. For each investment profile $x$, there exists a constant profile $\sigma$ such that $\mathbb{L}(x)=\mathbb{L}(\sigma)$ and $\mathbb{V}(x) \leq \mathbb{V}(\sigma)$.

Without loss of generality, assume that $x$ is a non-constant investment profile. If $\mathbb{L}^{t}$, the expected length of the hierarchy from period $t$ on, is constant, we can obviously choose a constant investment profile with the same expected length. If there exist two periods $t$ and $t^{\prime}$ such that $\mathbb{L}^{t}<\mathbb{L}^{t^{\prime}}$ then, by concavity of $\mathbb{L}^{*}$, we can marginally increase investment in $t$ and decrease investments in $t^{\prime}$ such that the expected length is fixed, but expected invested investment decreases.

Step 3. For each equilibrium profile $x$, there exists a constant equilibrium profile $\sigma$ such that $\mathbb{L}(x)=\mathbb{L}(\sigma)$.

Let $x$ be an equilibrium profile. By Step 2, it follows that there exists a constant profile with the same expected length and lower or equal expected investments. Consider the transfer 
scheme for which $t_{(i+1) i}=\beta$, for $i=0, \ldots$, and $t_{i j}=0$ otherwise. We now claim that we can choose $\beta \leq 1$ such that the investment in the constant profile is optimal for each agent. By contradiction, suppose that is not the case. Then, to choose the investment, each agent would need a (hypothetical) amount greater than 1 in return for finding the follower. But this contradicts that it was possible to incentivize the original investment. It is clear that the socially optimal transfer scheme sets $\beta=1$.

\section{Examples}

The aim of this section is to provide numbers for some of the profiles and schemes introduced above. For that matter, we consider the same technology as in Remark 1. Formally, let $p: \mathbb{R} \rightarrow[0,1]$ be the technology defined by

$$
p\left(x_{i}\right)=\frac{\sqrt{x_{i}}}{\frac{1}{4}+\sqrt{x_{i}}} .
$$

\subsection{First-best optimal investment profile}

By Theorem 2, we know that the first-best optimal investment profile is constant. Then, if at (2) we impose $x_{i}=x_{j}=\sigma$, for each $i, j \in \mathbb{N}_{0}$, we obtain

$$
\mathbb{V}(x)=\mathbb{V}(\sigma, \sigma, \ldots)=(1-\sigma) \sum_{n=0}^{+\infty}(p(\sigma))^{n}=\frac{1-\sigma}{1-p(\sigma)} .
$$

The value of $\sigma$ maximizing that expression is obtained by solving the first order condition

$$
\frac{1-\sigma}{1-p(\sigma)}=\frac{1}{p^{\prime}(\sigma)}
$$

Equivalently,

$$
(1-\sigma) \frac{1 / 4}{2 \sqrt{\sigma}(1 / 4+\sqrt{\sigma})^{2}}=(1-\sigma) p^{\prime}(\sigma)=1-p(\sigma)=\frac{1 / 4}{1 / 4+\sqrt{\sigma}}
$$

or

$$
\sqrt{\sigma}+6 \sigma-2=0
$$

whose solution is $\sigma^{*}=1 / 4$. Note that $p(1 / 4)=2 / 3$ and $\mathbb{V}(1 / 4,1 / 4, \ldots)=9 / 4$.

\subsection{The (second-best) socially optimal scheme}

Let us now consider the decentralized framework. We start with the full transfer predecessor scheme, which, as shown by Theorem 4, is the (second-best) socially optimal scheme. Formally, 
$t$ is defined such that $t_{i(i-1)}=1$, for each $i \geq 1, t_{00}=1$, and $t_{i j}=0$ otherwise. Then, each agent $i$ selects $x_{i}$ to maximize $p\left(x_{i}\right)-x_{i}$, whose first order condition is $p^{\prime}\left(x_{i}\right)=1$. Equivalently,

$$
1=8 \sqrt{x_{i}}\left(1 / 4+\sqrt{x_{i}}\right)^{2}
$$

whose unique solution is $\sigma^{\star}=0.1216$. As all agents are facing the same first-order condition, the optimal profile is precisely $\left(\sigma^{\star}, \sigma^{\star}, \ldots\right)$. Note that $p\left(x^{\star}\right)=0.582$ and $\mathbb{V}\left(x^{\star}, x^{\star}, \ldots\right) \approx 2.1$. One can infer from here that the price of anarchy for this scheme is small (about $1.07=2.25 / 2.1$ ).

\subsection{The initiator-optimal schemes}

Let now $t^{\alpha}$ be the $\alpha$-transfer initiator scheme, characterized in Theorem 3 as the initiatoroptimal schemes. Formally, for each $\alpha \in[0,1], t^{\alpha}$ is defined by $t_{(i+1) i}^{\alpha}=\alpha, t_{i 0}^{\alpha}=1-\alpha$, and $t_{i j}^{\alpha}=0$ otherwise. Then, each agent $i \geq 1$ selects $x_{i}$ to maximize $p\left(x_{i}\right) \alpha-x_{i}$, whose first order condition is $p^{\prime}\left(x_{i}\right)=1 / \alpha$. Equivalently,

$$
\alpha=8 \sqrt{x_{i}}\left(1 / 4+\sqrt{x_{i}}\right)^{2}
$$

whose solution (which cannot be explicitly obtained) is denoted by $x_{\alpha}^{*}$. Some numerical values are the following:

\begin{tabular}{|l|l|}
\hline$\alpha$ & $x_{\alpha}^{*}$ \\
\hline 0 & 0 \\
\hline 0.2 & 0.02357 \\
\hline 0.25 & 0.03 \\
\hline 0.26 & 0.0316 \\
\hline 0.265 & 0.0322336 \\
\hline 0.2655 & 0.0322998 \\
\hline 0.266 & 0.032366 \\
\hline 0.4 & 0.04984 \\
\hline 0.5 & 0.0625 \\
\hline 1 & 0.1216 \\
\hline
\end{tabular}

Now, the initiator aims to select $x_{0}$ to maximize

$$
\left(1-x_{0}\right)+p\left(x_{0}\right)+p\left(x_{0}\right) p\left(x_{\alpha}^{*}\right)(1-\alpha)+p\left(x_{0}\right)\left(p\left(x_{\alpha}^{*}\right)\right)^{2}(1-\alpha)+\cdots
$$


That is,

$$
\begin{aligned}
\left(1-x_{0}\right)+p\left(x_{0}\right)+p\left(x_{0}\right)(1-\alpha)\left(\sum_{n=1}^{\infty}\left(p\left(x_{\alpha}^{*}\right)\right)^{n}\right) & =\left(1-x_{0}\right)+p\left(x_{0}\right)+p\left(x_{0}\right)(1-\alpha) \frac{p\left(x_{\alpha}^{*}\right)}{1-p\left(x_{\alpha}^{*}\right)} \\
& =\left(1-x_{0}\right)+p\left(x_{0}\right)\left(1+(1-\alpha) \frac{p\left(x_{\alpha}^{*}\right)}{1-p\left(x_{\alpha}^{*}\right)}\right) \\
& =\left(1-x_{0}\right)+p\left(x_{0}\right)\left(\frac{1-p\left(x_{\alpha}^{*}\right)+(1-\alpha) p\left(x_{\alpha}^{*}\right)}{1-p\left(x_{\alpha}^{*}\right)}\right) \\
& =\left(1-x_{0}\right)+p\left(x_{0}\right)\left(\frac{1-\alpha p\left(x_{\alpha}^{*}\right)}{1-p\left(x_{\alpha}^{*}\right)}\right)
\end{aligned}
$$

Let

$$
A(\alpha)=\frac{1-\alpha p\left(x_{\alpha}^{*}\right)}{1-p\left(x_{\alpha}^{*}\right)} .
$$

Then, the initiator selects her investment $x_{0}$ so that she maximizes

$$
\left(1-x_{0}\right)+p\left(x_{0}\right) A(\alpha) .
$$

Thus, $\bar{x}_{\alpha}$ is the solution to

$$
p^{\prime}\left(x_{0}\right)=1 / A(\alpha)=\frac{1-p\left(x_{\alpha}^{*}\right)}{1-\alpha p\left(x_{\alpha}^{*}\right)} .
$$

Equivalently, $\bar{x}_{\alpha}$ is the solution to

$$
\frac{1}{4}+(1+\alpha) \sqrt{x_{\alpha}^{*}}=2 \sqrt{x_{0}}\left(\frac{1}{4}+\sqrt{x_{0}}\right)^{2} .
$$

Some numerical values are the following:

\begin{tabular}{|l|l|}
\hline$\alpha$ & $\bar{x}_{\alpha}$ \\
\hline 0 & 0.22588 \\
\hline 0.2 & 0.36051 \\
\hline 0.25 & 0.38312 \\
\hline 0.26 & 0.3875 \\
\hline 0.265 & 0.38968 \\
\hline 0.2655 & 0.3899 \\
\hline 0.266 & 0.39012 \\
\hline 0.4 & 0.44597 \\
\hline 0.5 & 0.48576 \\
\hline 1 & 0.67726 \\
\hline
\end{tabular}


Then,

$$
\begin{aligned}
\mathbb{E}_{0}\left(t^{1},\left(\bar{x}_{1}, x_{1}^{*}, x_{1}^{*}, \ldots\right)\right) & =\left(1-\bar{x}_{1}\right)+p\left(\bar{x}_{1}\right)\left(\frac{1-p\left(x_{1}^{*}\right)}{1-p\left(x_{1}^{*}\right)}\right) \\
& \approx(1-0.67726)+p(0.67726) \\
& \approx 1.0897 .
\end{aligned}
$$

Similarly,

$$
\begin{aligned}
\mathbb{E}_{0}\left(t^{0},\left(\bar{x}_{0}, x_{0}^{*}, x_{0}^{*}, \ldots\right)\right) & =\left(1-\bar{x}_{0}\right)+p\left(\bar{x}_{0}\right)\left(\frac{1}{1-p\left(x_{0}^{*}\right)}\right) \\
& \approx(1-0.22588)+p(0.22588) \\
& \approx 1.429419 .
\end{aligned}
$$

In general,

$$
\Phi(\alpha)=\mathbb{E}_{0}\left(t^{\alpha},\left(\bar{x}_{\alpha}, x_{\alpha}^{*}, x_{\alpha}^{*}, \ldots\right)\right)=\left(1-\bar{x}_{\alpha}\right)+p\left(\bar{x}_{\alpha}\right)\left(\frac{1-\alpha p\left(x_{\alpha}^{*}\right)}{1-p\left(x_{\alpha}^{*}\right)}\right)
$$

Thus, as argued in the Appendix, the optimal value is reached around $\alpha=0.2655$. Some numerical values are the following:

\begin{tabular}{|l|l|}
\hline$\alpha$ & $\Phi(\alpha)$ \\
\hline 0 & 1.429419 \\
\hline 0.2 & 1.692389 \\
\hline 0.25 & 1.699306 \\
\hline 0.26 & 1.701201 \\
\hline 0.265 & 1.7012544 \\
\hline 0.2655 & 1.7012545 \\
\hline 0.266 & 1.701253 \\
\hline 0.4 & 1.671493 \\
\hline 0.5 & 1.618237 \\
\hline 1 & 1.089735 \\
\hline
\end{tabular}

\subsection{Full transfer pre-predecessor}

Finally, let $t$ be the full transfer pre-predecessor transfer scheme considered in Remark 1 . Formally, $t$ is such that $t_{i(i-2)}=1$, for each $i \geq 2, t_{00}=t_{11}=1$, and $t_{i j}=0$ otherwise. As mentioned in Remark 1 , the profile $(1 / 16,1 / 16, \ldots)$ is an equilibrium and it satisfies $p(1 / 16)=$ $1 / 2$. Then, $\mathbb{V}(1 / 16,1 / 16, \ldots)=15 / 8$. 


\section{Discussion}

We have analyzed a stylized model on the optimal management of evolving hierarchies. In our model, an initiator invests into finding a subordinate, who will bring revenues to the joint venture and who will invest herself into finding another subordinate, and so on. The initiator sets a transfer scheme specifying how revenues are reallocated, via upward transfers, as the hierarchy evolves.

We believe our model is interesting on its own, but we have also argued that it has potentially important applications, relating to diverse areas such as network induction interventions, respondent-driven sampling, strategic recruiting in ongoing hierarchies, search and organizational hierarchy incentive tree mechanisms, or resource allocation in the presence of a hierarchical structure.

We have shown first that the socially optimal investment profile is unique and constant among members of the hierarchy. As for the decentralized approach to the model, we have considered two optimality notions for transfer schemes: initiator-optimal and socially-optimal schemes. We have shown that the former are schemes imposing to each member a full transfer to two recipients (the predecessor and the initiator) with a constant ratio among the transfers. We have also shown that the latter are schemes imposing full transfers to the immediate predecessors.

Our model has concentrated on the linear-hierarchy case, but the whole analysis can be smoothly generalized to account for branch hierarchies, i.e., situations in which a given agent can have more than one immediate subordinate.

An avenue for further research would be to extend the analysis in this paper beyond hierarchies containing a single highest-ranked agent (initiator). It is often the case that a given agent has more than one superior. For instance, for social mobilization schemes, an agent may be approached by several recruiters and may solve tasks for all of them. Similarly, two firms may jointly own an entity on an equal partnership basis and that entity may again own other entities, either alone or as joint ventures. Our decentralized notion of initiator optimality would have to be redefined for this setting. Other interesting issues for the management of these general hierarchies would also arise. 


\section{Appendix}

Let $f: \mathbb{R}_{+} \rightarrow \mathbb{R}_{+}$be such that, for each $z \in \mathbb{R}_{+}$,

$$
f(z)=\sqrt{z}\left(\frac{1}{4}+\sqrt{z}\right)^{2} .
$$

Note that, for each $\alpha \in[0,1], x_{\alpha}^{*}$ is the solution to

$$
f(z)=\frac{\alpha}{8}
$$

whereas $\bar{x}_{\alpha}$ is the solution to

$$
f(z)=\frac{1}{8}+\frac{1+\alpha}{2} \sqrt{x_{\alpha}^{*}} .
$$

Thus, it all amounts to solve equations of the form

$$
f(z)=A,
$$

for some scalar $A$.

Let $y=\sqrt{z}$. Then,

$$
f(z)=\frac{A}{16} \Longleftrightarrow 16 y^{3}+8 y^{2}+y-A=0 .
$$

Then, $y$ could be explicitly obtained by the so-called cubic formula, first published by Cardano (1545). More precisely,

$$
\begin{aligned}
y & =\left(\left(\frac{-b^{3}}{27 a^{3}}+\frac{b c}{6 a^{2}}-\frac{d}{2 a}\right)+\left(\left(\frac{-b^{3}}{27 a^{3}}+\frac{b c}{6 a^{2}}-\frac{d}{2 a}\right)^{2}+\left(\frac{c}{3 a}-\frac{b^{2}}{9 a^{2}}\right)^{3}\right)^{1 / 2}\right)^{1 / 3} \\
& +\left(\left(\frac{-b^{3}}{27 a^{3}}+\frac{b c}{6 a^{2}}-\frac{d}{2 a}\right)-\left(\left(\frac{-b^{3}}{27 a^{3}}+\frac{b c}{6 a^{2}}-\frac{d}{2 a}\right)^{2}+\left(\frac{c}{3 a}-\frac{b^{2}}{9 a^{2}}\right)^{3}\right)^{1 / 2}\right)^{1 / 3}-\frac{b}{3 a}
\end{aligned}
$$

where $a=16, b=8, c=1$ and $d=-A$.

Now,

$$
\left(\frac{-b^{3}}{27 a^{3}}+\frac{b c}{6 a^{2}}-\frac{d}{2 a}\right)=\frac{1+54 A}{1728}=\frac{1+54 A}{2^{6} \cdot 3^{3}},
$$

and

$$
\left(\frac{c}{3 a}-\frac{b^{2}}{9 a^{2}}\right)=-\frac{1}{144}=-\frac{1}{2^{4} \cdot 3^{2}} .
$$

Then,

$$
\left(\frac{-b^{3}}{27 a^{3}}+\frac{b c}{6 a^{2}}-\frac{d}{2 a}\right)^{2}+\left(\frac{c}{3 a}-\frac{b^{2}}{9 a^{2}}\right)^{3}=\frac{(1+54 A)^{2}-1}{2^{12} \cdot 3^{6}}=\frac{(27 A+1) A}{2^{10} \cdot 3^{3}}
$$


From here, it follows that

$$
\begin{aligned}
y & =\left(\frac{1+54 A}{2^{6} \cdot 3^{3}}+\left(\left(\frac{1+54 A}{2^{6} \cdot 3^{3}}\right)^{2}+\left(-\frac{1}{2^{4} \cdot 3^{2}}\right)^{3}\right)^{1 / 2}\right)^{1 / 3} \\
& +\left(\frac{1+54 A}{2^{6} \cdot 3^{3}}-\left(\left(\frac{1+54 A}{2^{6} \cdot 3^{3}}\right)^{2}+\left(-\frac{1}{2^{4} \cdot 3^{2}}\right)^{3}\right)^{1 / 2}\right)^{1 / 3}-\frac{1}{6} \\
& =\left(\frac{1+54 A}{2^{6} \cdot 3^{3}}+\left(\frac{54 A(54 A+2)}{2^{12} \cdot 3^{6}}\right)^{1 / 2}\right)^{1 / 3} \\
& +\left(\frac{1+54 A}{2^{6} \cdot 3^{3}}-\left(\frac{54 A(54 A+2)}{2^{12} \cdot 3^{6}}\right)^{1 / 2}\right)^{1 / 3}-\frac{1}{6} \\
& \left.=\left(\frac{1+54 A+\sqrt{54 A(54 A+2)}}{2^{6} \cdot 3^{3}}\right)^{1 / 3}\right)^{1 / 3}-\frac{1}{6} \\
& +\left(\frac{1+54 A-\sqrt{54 A(54 A+2)}}{2^{6} \cdot 3^{3}}+\sqrt[3]{1+54 A-\sqrt{54 A(54 A+2)}}-2\right) \\
& =\frac{1}{12(\sqrt[3]{1+54 A+\sqrt{54 A(54 A+2)}})}
\end{aligned}
$$

Note that we could get complex numbers in the expression above. We restrict ourselves to the real solutions, i.e., $A(54 A+2)>0$ or, equivalently, $A \in(-\infty,-1 / 27] \cup[0,+\infty){ }^{4}$

Let $g:(-\infty,-1 / 27] \cup[0,+\infty) \rightarrow \mathbb{R}$, such that, for each $A \in(-\infty,-1 / 27] \cup[0,+\infty)$,

$$
g(A)=\frac{1}{12}(\sqrt[3]{1+54 A+\sqrt{54 A(54 A+2)}}+\sqrt[3]{1+54 A-\sqrt{54 A(54 A+2)}}-2) .
$$

Then, a solution to the equation

$$
f(z)=\frac{A}{16}
$$

is

$$
z=(g(A))^{2}
$$

Note that

$$
g(1)=\frac{1}{12}(\sqrt[3]{55+\sqrt{3024}}+\sqrt[3]{55-\sqrt{3024}}-2)=\frac{1}{4}
$$

as expected. ${ }^{5}$

We can then state that

$$
x_{\alpha}^{*}=(g(2 \alpha))^{2}=\frac{1}{144}(\sqrt[3]{1+108 \alpha+6 \sqrt{6 \alpha(54 \alpha+1)}}+\sqrt[3]{1+108 \alpha-6 \sqrt{6 \alpha(54 \alpha+1)}}-2)^{2},
$$

\footnotetext{
${ }^{4}$ In our cases, $A$ will always be positive.

${ }^{5}$ Recall we mentioned above that $x_{1 / 2}^{*}=1 / 16$.
} 
whereas

$$
\bar{x}_{\alpha}=\left(g\left(2+8(1+\alpha) \sqrt{x_{\alpha}^{*}}\right)\right)^{2} .
$$

For each $\alpha \in[0,1]$, we could then define the function $\Phi:[0,1] \rightarrow \mathbb{R}$ such that

$$
\Phi(\alpha)=\mathbb{E}_{0}\left(t^{\alpha},\left(\bar{x}_{\alpha}, x_{\alpha}^{*}, x_{\alpha}^{*}, \ldots\right)\right)=\left(1-\bar{x}_{\alpha}\right)+p\left(\bar{x}_{\alpha}\right)\left(\frac{1-\alpha p\left(x_{\alpha}^{*}\right)}{1-p\left(x_{\alpha}^{*}\right)}\right) .
$$

The goal is to find

$$
\alpha^{*}=\arg \max _{\alpha \in[0,1]} \Phi(\alpha) .
$$

Note that, as mentioned above, $\alpha^{*} \approx 0.2655$. 


\section{References}

[1] Aral, S., Walker, D., (2011) Creating Social Contagion Through Viral Product Design: A Randomized Trial of Peer Influence in Networks Management Science 57, 1623-1639.

[2] Bird, C., (1976), On cost allocation for a spanning tree: a game theoretic approach, Networks 6, 335-350.

[3] Cardano, G., (1545) Ars magna or The Rules of Algebra, Dover (published 1993).

[4] Claus, A., Kleitman, D., (1973), Cost allocation for a spanning tree, Networks 3, 289-304.

[5] Corominas-Murtra, B., Gonid, J., Sole R.V., Rodriguez-Caso, C., (2013) On the origins of hierarchy in complex networks. Proceedings of the National Academy of Sciences 110, 13316-13321.

[6] Dong, B., Ni, D., Wang, Y., (2012), Sharing a polluted river network, Environmental and Resource Economics 53, 367-387.

[7] Emek, Y., Karidi, R., Tennenholtz, M., Zohar, A., (2011), Mechanisms for multi-level marketing, Proc. of 12th ACM Conference on Electronic Commerce, 209-218.

[8] Goldlücke, S., (2017) Strategic recruiting in ongoing hierarchies Economics Letters 156, 176-178.

[9] Heckathorn D., (1997) Respondent-Driven Sampling: A New Approach to the Study of Hidden Populations. Social Problems 44, 174-199.

[10] Hougaard, J.L., (2018) Allocation in Networks. MIT Press. Cambridge, MA.

[11] Hougaard, J.L., Moreno-Ternero, J.D., Tvede, M., Østerdal, L.P., (2017) Sharing the proceeds from a hierarchical venture. Games and Economic Behavior 102, 98-110.

[12] Littlechild, S., Owen. G., (1973), A simple expression for the Shapley value in a special case, Management Science 20, 370-372.

[13] Lv, Y., Moscibroda, T., (2013), Fair and resilient incentive tree mechanisms. Proc. of the 2013 ACM symposium on Principles of distributed computing, 230-239.

[14] Mesarovic, M.D., Macko, D., Takahara, Y., (1970) Theory of Hierarchical, Multilevel, Systems. Academic Press. New York. 
[15] Mihm, J., Loch, C., Wilkinson, D., Huberman, B., (2010) Hierarchical Structure and Search in Complex Organizations. Management Science 56, 831-848

[16] Ni, D. an Y. Wang, (2007), Sharing a polluted river, Games and Economics Behavior, 60, 176-186.

[17] Nicolis, J.S., (2012) Dynamics of hierarchical systems: an evolutionary approach. Springer.

[18] Pickard, G., Pan, W., Rahwan, I., Cebrian, M., Crane, R., Madan, A., Pentland, A., (2011) Time critical social mobilization. Science 334, 509-512.

[19] Rivkin, J. W. (2000) Imitation of complex strategies. Management Science 46, 824-844.

[20] Salthe, S.N., (1985) Evolving hierarchical systems. Columbia University Press.

[21] Topkis, (1979) Equilibrium Points in Non-Zero Sum n-Person Submodular Games. SIAM J. of Control and Optimization, 17, 773-787.

[22] Valente, T.W., (2012) Network Interventions. Science 337, 49-53. 